\title{
Impactos psicossociais da estética dentária na qualidade de vida de pacientes submetidos a próteses: revisão de literatura
}

Psychosocial impacts of dental aesthetics on the quality of life of patients submitted to prostheses: literature review Impactos psicosociales de la estética dental en la calidad de vida de pacientes sometidos a prótesis: revisión de literatura

\author{
Jaqueline Oliveira BARRETO ${ }^{1}$ \\ Millena Lorrana de Almeida SOUSA ${ }^{1}$ \\ Silvestre Estrela da SILVA-JÚNIOR ${ }^{1}$ \\ Julliana Cariry Palhano FREIRE ${ }^{2}$ \\ Túlio Neves de ARAÚJO ${ }^{3}$ \\ George Borja de FREITAS ${ }^{3}$ \\ Eduardo DIAS-RIBEIRO ${ }^{4}$
}

${ }^{1}$ Universidade Federal de Campina Grande, Departamento de Odontologia, Centro de Saúde e Tecnologia Rural (CSTR), 58708-110 Patos, Paraíba, Brasil ${ }^{2}$ Universidade Estadual da Paraíba, Departamento de Odontologia, 58429-500 Campina Grande - PB, Brasil

${ }^{3}$ Faculdades Integradas de Patos, Departamento de Odontologia, Centro de Ciências Biológicas e da Saúde (CCBS) 58429-500 Patos, Paraíba, Brasil

${ }^{4}$ Universidade Federal da Paraíba, Departamento de Clínica e Odontologia Social, Centro de Ciências da Saúde (CCS), 58051-900 João Pessoa-PB, Brasil

\section{Resumo}

Introdução: Atualmente, os índices do edentulismo diminuíram, embora a preocupação pela reabilitação dentária aumentaram, o que gera foco de estudos sobre as expectativas que essa população procura os serviços dessa área da odontologia. Objetivo: O objetivo deste estudo foi fazer uma revisão de literatura sobre os impactos psicossociais que a estética dentária pode causar na qualidade de vida de sujeitos que são submetidos a próteses convencionais e sobre implantes. Método: Foram selecionados artigos disponíveis na base de dados MEDLINE (Medical Literature Analysis and Retrieval System on-line) publicados em inglês, no período compreendido entre 2008-2018; Resultados: Os principais resultados encontrados foi que a estética dentária tem influência direta na autoestima e por isso os indivíduos sofrem com uma barreira pessoal e profissional, manifestando negativamente na sua qualidade de vida. Porém, população que são reabilitados com próteses sobre implantes parece ter maior preocupação com sua estética e por isso sofreram mais com depressão, ao passo que isso aumenta quando se trata de dentes posicionados anteriormente na arcada dentária. Conclusão: Conclui-se que a estética dentária influencia psicossocialmente em um indivíduo, causando impactos na qualidade de vida de edêntulos, além disso, há diferenças psicológicas na população que busca reabilitação com próteses convencionais e implantossuportadas e a posição que os dentes perdidos ocupavam no arco dentário.

Descritores: Próteses e Implantes; Estética Dentária; Qualidade de Vida.

\section{Abstract}

Introduction: Currently, edentulism indexes have decreased, although the concern for dental rehabilitation has increased, which generates a focus of studies on the expectations that this population seeks the services of this area of dentistry. Objective: The objective of this study was to review the literature on the psychosocial impacts that dental aesthetics can cause on the quality of life of people who are submitted to conventional prostheses and implants. Method: Selected articles were published in MEDLINE (Medical Literature Analysis and Retrieval System on-line) published in English, in the period between 2008-2018; Results: The main results were that dental aesthetics have a direct influence on self-esteem and therefore individuals suffer from a personal and professional barrier, manifesting negatively in their quality of life. However, people who are rehabilitated with implant prostheses seems to have greater concern for their aesthetic and therefore suffered more from depression, while it increases when it comes to teeth previously positioned in dental arch. Conclusion: It is concluded that dental aesthetics influence psychosocially in an individual, causing impacts on the quality of life of edentulous, in addition, there are psychological differences in the population that seeks rehabilitation with conventional prostheses and implant-supported and the position that the lost teeth occupied in the dental arch.

Descriptors: Prostheses and Implants; Esthetics, Dental; Quality of Life.

\section{Resumen}

Introducción: Actualmente, los índices del edentulismo disminuyeron, aunque la preocupación por la rehabilitación dental aumentó, lo que genera foco de estudios sobre las expectativas que esa población busca los servicios de esa área de la odontología. Objetivo: El objetivo de este estudio fue hacer una revisión de literatura sobre los impactos psicosociales que la estética dental puede causar en la calidad de vida de sujetos que son sometidos a prótesis convencionales y sobre implantes. Método: Se han seleccionado artículos disponibles en la base de datos MEDLINE (Diagnóstico de la literatura en tiempo real) publicados en inglés durante el período comprendido entre 2008-2018; Resultados: Los principales resultados encontrados fueron que la estética dental tiene influencia directa en la autoestima y por eso los individuos sufren con una barrera personal y profesional, manifestando negativamente en su calidad de vida. Sin embargo, la población que es rehabilitada con prótesis sobre implantes parece tener mayor preocupación con su estética y por lo que sufren más con depresión, mientras que esto aumenta cuando se trata de dientes colocados anteriormente en la arcada dental. Conclusión: Se concluye que la estética dental influye psicosocialmente en un individuo, causando impactos en la calidad de vida de edentulous, además, hay diferencias psicológicas en la población que busca rehabilitación con prótesis convencionales e implantosoportadas y la posición que los dientes perdidos ocupaban en el arco dental.

Descriptores: Prótesis e Implantes; Estética Dental; Calidad de Vida.

\section{INTRODUÇÃO}

Ao longo das últimas décadas, o termo qualidade de vida vem tomando destaque para a Organização Mundial de Saúde (OMS), por sua influência na saúde geral de qualquer indivíduo, já que só pode ser considerado totalmente saudável o indivíduo com bem estar físico e psicológico. Nessa perspectiva, é insuficiente para a Odontologia Reabilitadora considerar apenas o aspecto funcional, devendo, portanto, incluir o bem estar psicossocial, diretamente relacionado com a estética dentária do paciente $^{1-2}$

A perda da estética dos dentes vem mostrando reflexos negativos nas interações sociais, como seu menor desempenho no ambiente de trabalho ou escola. Isso acontece devido a sociedade pregar o sorriso como umas das principais características estética, o que causa satisfação ou não ao indivíduo combinado com o recebimento de um tratamento pode resultar no insucesso, revelando-se como um problema potencialmente significante ${ }^{1,3-4}$. 
Dentre os tratamentos de reabilitação oral, o implante dentário e a prótese removível são os mais indicados e que mostram altas taxas de sucesso, exercendo um papel fundamental para a melhoria da saúde bucal por devolver a função de mastigação, fonação e estética. Dessas duas, a terapia de implantes é atualmente um tipo de cirurgia oral mais requisitada para recuperar áreas edêntulas parcial ou total por dar uma sensação de maior semelhança ao dente perdido, naturalidade e melhor higiene. Mas, esse procedimento financeiramente ainda não é tão acessível $^{5-6}$.

Portanto, conhecer as percepções e expectativas de cada paciente é essencial para conseguir o sucesso total após o tratamento, bem como investigar sua satisfação com os resultados conquistados, levando aos profissionais de saúde a se preocuparem não só com a integridade física, mas também com a mental e a qualidade de vidas de quem procura os seus serviços, permitindo que ambos os lados sejam beneficiados. ${ }^{4}$ Assim, o objetivo deste estudo foi realizar uma revisão de literatura sobre os impactos da estética dentária em pacientes que se submeteram a reabilitação com prótese fixa ou removível, bem como seus efeitos na qualidade de vida.

\section{MATERIAL E MÉTODO}

Para a elaboração do presente trabalho as seguintes etapas foram percorridas: estabelecimento da hipótese e objetivos do estudo; estabelecimento de critérios de inclusão e exclusão de artigos (seleção da amostra). Tomando como guiada revisão, formulouse a seguinte questão: a estética dentária pode causar impactos psicossociais e na qualidade de vida de pacientes submetidos a próteses convencionais e sobre implantes?

Os artigos foram selecionados utilizando a base de dados MEDLINE (Medical Literature Analysis and Retrieval Sistem on-line). As estratégias utilizadas para localizar os artigos tiveram como eixo norteador a pergunta e os critérios de inclusão da revisão, previamente estabelecidos para manter a coerência na busca dos artigos e evitar possíveis vieses. As palavras-chave utilizadas foram os descritores "prostheses and implants", "esthetics dental" e "quality of life", acordando com o Decs.

Os critérios de inclusão foram artigos publicados em inglês, com os resumos disponíveis no MEDLINE, no período compreendido entre 20082018; artigos cuja metodologia adotada permitisse obter evidências fortes, tais como estudos randomizados controlados ou não randomizados e estudos empíricos. Foram excluídos estudos que não comparassem os impactos da estética dentária entre os sujeitos reabilitados com próteses convencionais e próteses fixas. Por fim, através da busca de acesso on-line, a amostra final resultou em 20 artigos.

\section{REVISÃO DA LITERATURA}

Nas últimas décadas, o interesse pelas variações emocionais sofridas pelos indivíduos tem crescido mundialmente dentro da atividade odontológica, tendo em vista a necessidade do acolhimento pelo profissional do estado psicológico dos pacientes, valorizando a função e a estética, assim como também assegurando sua qualidade de vida $^{7-8}$.

O edentulismo, caracterizado pela perda total ou parcial dos dentes, está presente em pessoas de todas as faixas etárias. Logo, não é uma condição associada ao envelhecimento, mas tem como principais causas as lesões cariosas e doença periodontal. Na busca pelo restabelecimento da função e estética e com o intuito de melhorar a qualidade de vida desses indivíduos, a Odontologia moderna lança mão do uso de próteses fixas ou removíveis indicadas pelo profissional de acordo com a necessidade do paciente ${ }^{9}$.

O termo qualidade de vida é definido como a percepção que o indivíduo tem de si mesmo e dentro da sociedade, suas expectativas, padrões de beleza e preocupações, e quando esses requisitos não são atendidos, pode ser gerado impactos com efeitos negativos na vida cotidiana das pessoas, como por exemplo o transtorno de ansiedade, baixa autoestima, insegurança, intolerância social e depressão ${ }^{1,10}$.

Segundo Rotundo et al. ${ }^{11}$ a estética dos dentes pode não influenciar apenas na autoestima, mas também em relacionamentos afetivos, desempenho profissional, oportunidades sociais e de emprego, o que reflete significativamente na qualidade de vida. $\mathrm{O}$ que se confirma no estudo de Chen et al. ${ }^{5}$, quando buscaram comparar traços psicossociais da estética dentária pré e pós-terapia de implantes, observando o comprometimento na vida social e na autoestima antes do tratamento, seguindo para uma melhora nos impactos de interação social, atitudes estéticas, autoconfiança e qualidade de vida depois da reabilitação.

Entretanto para Clijmans et al. ${ }^{12}$, a condição estética bucal ocorre de forma particular em cada sujeito, sendo por isso que para alguns comprometidos não é afetado psicologicamente em nenhuma forma pelo problema, enquanto para outros, barreira ao sucesso pessoal e social pode ser automaticamente experimentados.

Quanto ao tipo de reabilitação oral, no estudo de Cardoso et al. ${ }^{13}$ que investigaram a qualidade de vida relacionada a saúde bucal e a eficiência mastigatória em pacientes tratados com próteses dentária convencionais e implantes dentário, concluíram que o grupo reabilitado com próteses fixas mostrou melhor qualidade de vida, maior satisfação e maior eficiência mastigatória quando comparada com os que receberam a prótese removível. 
Quando se fala em qualidade de vida e bemestar mental e social, a questão de como o indivíduo se ver é imposta e se relaciona diretamente com a forma como lida e domina o ambiente e como é afetado pelas reações que os outros direciona para si mesmo provocando uma mudança de autoestima e consequentemente na qualidade de vida ${ }^{1,3,4}$.

Logo esse estado é relativamente instável e a literatura ainda não consegue afirmar se ela pode ser melhorada com o tratamento protético por insuficiência de estudos sobre a mesma. O que não é satisfatório para psicologia aplicada à odontologia, pois segundo Perillo et al. ${ }^{14}$, pessoas com maior qualidade de vida têm maior autoestima, são mais felizes, mais satisfeitas com suas vidas, menos deprimidos, mais competente no trabalho e mais saudáveis físico e mentalmente.

Isso fica evidente quando Clijmans et al. ${ }^{12}$ chegaram a uma associação estatisticamente significante através de questionários que avalia a autoestima (escala de Rosenberg) e qualidade de vida (OHIP-14). Esses autores perceberam que quando um aumentava, o outro diminuía, sugerindo que pessoas com maior autoestima também tinha melhores qualidade de vida. $\mathrm{O}$ que se assemelhou com os estudos de Patel et al. ${ }^{15}$, Torres et al. ${ }^{16}$, Al-Omiri et al. ${ }^{17}$ e Estevan et al. ${ }^{18}$.

Em contrapartida no trabalho de Komagamine et al. ${ }^{19}$ foi visto que a maior parte dos pacientes que optam pelo uso das próteses removíveis apresentam menos preocupação em relação à estética, e julgam a aparência e a retenção suficiente da prótese como o fator que mais influencia na satisfação com a saúde bucal e na qualidade de vida.

Conforme Fava et al. ${ }^{2}$ não é mais suficiente simplesmente restaurar um espaço edêntulo por um dente funcional se não levar em conta todo o transtorno psicológico gerado quando não se atinge a satisfação do paciente. Portanto considerar a percepção e os anseios dos pacientes no préoperatório de cirurgias de implantes, ou antes, de uma confecção de uma prótese é fundamental para garantir a melhora na qualidade de vida do paciente no futuro ${ }^{4}$.

Um estudo anterior revelou que a demanda por próteses dentárias provavelmente será solicitada se o dente superior ou anterior estiver ausente ${ }^{20}$. O apelo estético anterior pode ajudar o corpo a estabelecer atratividade e construir auto-estima e felicidade $^{21}$. Pelo contrário, decaído os dentes anteriores mostraram ter impacto negativo nas percepções de atratividade facial $^{22}$.

\section{DISCUSSÃO}

O edentulismo ainda é uma condição de saúde bucal presente na atualidade, mesmo esta mostrando redução dos seus índices. A posição que o elemento dentário ocupava na arcada dentária causa transtornos psicológicos e funcionais diferentes para um indivíduo, tendo que os dentes anteriores influenciam diretamente na estética, os posteriores, na função e ambos os posicionamentos se relacionam com a qualidade de vida ${ }^{7,23-26}$.

A literatura mostra que a reabilitação com próteses melhora significativamente a qualidade de vida do indivíduo fisicamente e psicologicamente, e isso pode aumentar dependendo se forem removíveis ou suportadas sobre implantes s,27-36. $^{5}$.

Além disso, fica claro que a perda de elementos dentários pode causar impactos psicossociais que influencia na autoestima dos sujeitos, refletindo seriamente na sua vida pessoal e profissional, consequentemente na sua qualidade de vida $^{1,3,4,15-18}$.

Entretanto, estudos mostram que pacientes podem responder psicologicamente diferente dos demais quanto à perda de dentes, a ponto de isso não influenciar de nenhuma maneira em sua qualidade de vida $^{12,19}$. O que se deve investigar nesses estudos é se os pacientes que se comportaram dessa forma perderam apenas dentes anteriores e uma comparação destes grupos com sujeitos que perderam apenas dentes posteriores seria de alto interesse.

A expectativa do paciente frente a qualquer tipo de tratamento, principalmente estético, deve ser considerada como um fator importante para o resultado final, o que pode leva-lo a construir expectativas, que quando não superadas causa insatisfação em relação ao tratamento ${ }^{2,4}$.

\section{CONCLUSÃO}

A estética dentária influencia

psicossocialmente em um indivíduo, de forma que a perda dos dentes causa impactos negativos e piora sua qualidade de vida, afetando além da condição física e funcional, a sua autoestima. Foram observadas diferenças emocionais da população que busca reabilitação com próteses convencionais e implantossuportadas, sendo a primeira menos preocupada esteticamente.

\section{REFERÊNCIAS}

1. Gavric A, Mirceta D, Jakobovic M, Pavlic A, Zrinski MT, Spalj S. Cranio dento facial characteristics, dental esthetics-related quality of life, and self-esteem. Am J Orthod Dentofacial Orthop. 2015;147(6):711-18.

2. Fava J, Lin M, Zahran M, Jokstad A. Single implant-supported crowns in the aesthetic zone: patient satisfaction with aesthetic appearance compared with appraisals by laypeople and dentists. Clin Oral implants Res. 2015;26(10): 1113-20.

3. de Couto Nascimento V, de Castro Ferreira Conti AC, de Almeida Cardoso M, Valarelli DP, de Almeida-Pedrin RR. Impact of orthodontic 
treatment on self-esteem and quality of life of adult patients requiring oral rehabilitation. Angle Orthod. 2016;86(5):883-85.

4. Pereira CP, Abreu LG, Dickc BD, de Luca Canto G, Paiva SM, Flores-Mir C. Patient satisfaction after orthodontic treatment combined with orthognathic surgery: A systematic review. Angle Orthod. 2016;86(3):495-508.

5. Chen P, Yu S, Zhu G. The psychosocial impacts of implantation on the dental aesthetics of missing anterior teeth patients. $\mathrm{Br}$ Dent J. 2012; 213(11):E20

6. Benic GI, Wolleb K, Sancho-Puchades $\mathrm{M}$, Hämmerle $\mathrm{CH}$. Systematic review of parameters and methods for the professional assessment of aesthetics in dental implant research. Clin Oral implants Res. 2012; 39(Suppl 12):160-92.

7. Peñarrocha MA, Carrillo C, Boronat A, Martí EM. Level of satisfaction in patients with maxillary mull-arch fixed protheses: zigomatic versus convencional implants. Int $\mathbf{J}$ Oral Maxillofac Implants. 2007; 22(5):769-73.

8. Benson PE, Da'as T, Johal A, Mandall NA, Williams AC, Baker SR et al. Relationships between dental appearance, selfesteem, socioeconomic status, and oral healthrelated quality of life in UK schoolchildren: A 3-year cohort study. Eur J Orthod. 2015;37(5):481-90.

9. Alzarea B. Oral health related quality-of-life outcomes of partially edentulous patients treated with implant-supported single crowns or fixed partial dentures J Clin Exp Dent. 2017;9(5):666-71.

10.Frejmant MW, Vargas IA, Rösing CK, Closs LQ. Dentofacial deformities are associated with lower degrees of self-esteem and higher impact on oral health-related quality of life: results from na observational study involving adults. J Oral Maxillofac Surg.2013;71(4):763-67.

11.Rotundo R, Nieri M, Bonaccini $\quad D$, Mori M, Lamberti E, Massironi D et al. The Smile Esthetic Index (SEI): a method to measure the esthetics of the smile. an intra-rater and inter-rater agreement study. Eur J Oral Implantol.2015; 8(4):397-403.

12.Clijmans M, Lemiere J, Fieuws S, Willems G. Impact of self-esteem and personality traits on the association between orthodontic treatment need and oral health-related quality of life in adults seeking orthodontic treatment. Eur J Orthod. 2015;37(6):643-50.

13. Cardoso RG, Melo LA, Barbosa GA, Calderon PD, Germano AR, Mestriner W Junior et al. Impact of mandibular conventional denture and overdenture on quality of life and masticatory efficiency. Braz Oral Res. 2016;30(1):e102.

14.Perillo L, Esposito M, Caprioglio A, Attanasio S, Santini AC, Carotenuto M. Orthodontic treatment need for adolescents in the Campania region: the malocclusion impact on self-concept.

Patient Prefer Adherence. 2014;8:353-59.

15.Patel P, Brown S, Nazarian A. A better quality of life with implant-retained overdentures. Dent Today. 2012;31(1):156,158.

16. Torres BL, Costa FO, Modena CM, Cota LO, Côrtes MI, Seraidarian PI. Association between personality traits and quality oflife in patients treated with conventional mandibular dentures or implant-supported overdentures. J Oral Rehabil. 2011;38(6):454-61.

17.Al-Omiri MK, Hammad OA, Lynch E, Lamey PJ, Clifford TJ. Impacts of implant treatment on daily living. Int Oral Maxillofac Implants. 2011; 26(4):877-86.

18.Fernandez-Estevan LF, Selva-Otaolaurruchi EJ, Montero J, Sola-Ruiz F. Oral health-related quality of life of implant-supported overdentures versus conventional complete prostheses: Retrospective study of a cohort of edentulous patients. Med Oral Patol Oral Cir Bucal. 2015;20(4):e450-58.

19.Komagamine Y, Kanazawa M, Kaiba Y, Sato Y, Minakuchi S, Sasaki Y. Association between selfassessment of complete dentures and oral healthrelated quality of life. J Oral Rehabil. 2012; 39(11):847-57.

20.Mukatash GN, Al-Rousan M, Al-Sakarna B. Needs and demands of prosthetic treatment among two groups of individuals. Indian J Dent Res. 2010;21(4):564-67.

21.Gerritsen AE, Allen PF, Witter DJ, Bronkhorst EM, Creugers NH. Toothlossand oral healthrelated quality of life: a systematic review and meta-analysis. Health Qual Life Outcomes. 2010;8:126.

22.Kershaw S, Newton JT, Williams DM. The influence of tooth colour on the perception sofpersona lcharacteristics among female dental patients: comparisons of unmodified, decayedand 'whitened' teeth. Br Dent J. 2008;204(5):E9.

23.Towfighi PP, Brunsvold MA, Storey AT, Arnold RM, Willman DE, McMahan CA. Pathologic migration of anterior teeth in patients with moderate to severe periodontitis. J Periodontol. 1997;68(10):967-72.

24.Tatum RC, Tatum BM, Marfatia-Rege AT, Amant KS. Immediate esthetic treatment for anterior teeth: reportof cases. J Am Dent Assoc. 1989; 118(5):575-77.

25.Kapur A, Chawla HS, Goyal A, Gaube K. An esthetic point of view in very young children. $\mathbf{J}$ Clin Pediatr Dent.2005; 30(2):99-103.

26.Ponsi J, Lahti S, Rissanen H, Oikarinen K. Change in subjective oral healthafter single dental implanttreatment. Int J Oral Maxillofac Implants. 2011; 26(3):571-77. 
27. Shaw WC. Factors influencing the desire for orthodontic treatment. Eur J Orthod. 1981;3(3):151-62.

28. Sheats RD, McGorray SP, Keeling SD, Wheeler TT, King GJ. Occlusal traits and perceptionof orthodontic need in eighth grade students. Angle Orthod.1998;68(2):107-14.

29. Al-Omiri MK, Karasneh JA, Lynch E, Lamey PJ, Clifford TJ. Impacts of missin gupper anterior teeth on daily living. Int Dent J.2009;59(3):127-32.

30.Carlsson GE, Johansson A, Johansson AK, Ordell S, Ekbäck G, Unell L. Attitudes toward dental appearance in 50-and 60-year-old subjects living in Sweden. J Esthet Restor Dent. 2008; 20(1):46-55.

31.Xiao J, Zhou X, Zhu WD, Zhang B, Li JY, Xu X. The prevalence of tooth discolouration and the self-satisfaction with tooth colour in a Chinese urban population. J Oral Rehabil. 2007; 34(5):351-60.

32. Akarslan ZZ, Sadik B, Erten H, Karabulut E. Dental esthetic satisfaction, received and desired dental treatments for improvement of esthetics. Indian J Dent Res. 2009; 20(2):195-200.

33.Wang SW, Repetti RL, Campos B. Job stress andfamily social behavior: themoderating role ofneuroticism. J Occup Health Psychol. 2011; 16(4):441-56.

34. Samorodnitzky-Naveh GR, Geiger SB, Levin L. Patients' satisfactionwith dental esthetics. J Am Dent Assoc. 2007;138(6):805-8.

35. Hassel A, Wegener I, Rolko C, Nitschke I. Selfrating of satisfaction with dental appearance in anelderly German population. Int Dent J. 2008; 58(2):98-102.

36.Tin-Oo MM, Saddki N, Hassan N. Factors influencing patient satisfaction with dental appearance and treatments they desire to improve aesthetics. BMC Oral Health.2011;11:6.

\section{CONFLITO DE INTERESSES}

Os autores declaram não haver conflitos de interesse.

\section{AUTOR PARA CORRESPONDENCIA}

\section{Jaqueline Oliveira Barreto}

jacquinha_barreto@hotmail.com
Submetido em 17/07/2018

Aceito em 04/10/2018 